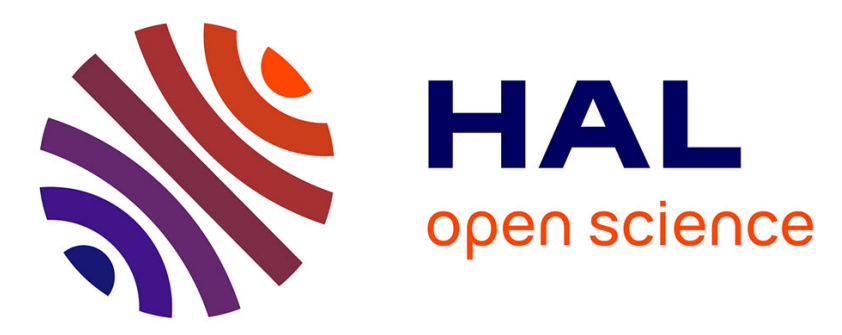

\title{
Quantitative modulation of polycomb silencing underlies natural variation in vernalization.
}

Vincent Coustham, Peijin Li, Amy Strange, Clare Lister, Jie Song, Caroline Dean

\section{- To cite this version:}

Vincent Coustham, Peijin Li, Amy Strange, Clare Lister, Jie Song, et al.. Quantitative modulation of polycomb silencing underlies natural variation in vernalization.. Science, 2012, 337 (6094), pp.584-587. 10.1126/science.1221881 . hal-02650183

\section{HAL Id: hal-02650183 \\ https://hal.inrae.fr/hal-02650183}

Submitted on 29 May 2020

HAL is a multi-disciplinary open access archive for the deposit and dissemination of scientific research documents, whether they are published or not. The documents may come from teaching and research institutions in France or abroad, or from public or private research centers.
L'archive ouverte pluridisciplinaire HAL, est destinée au dépôt et à la diffusion de documents scientifiques de niveau recherche, publiés ou non, émanant des établissements d'enseignement et de recherche français ou étrangers, des laboratoires publics ou privés. 


\title{
Quantitative Modulation of Polycomb Silencing Underlies Natural Variation in Vernalization
}

\author{
Vincent Coustham, ${ }^{*}$ Peijin Li, Amy Strange, + Clare Lister, Jie Song, Caroline Dean¥ \\ John Innes Centre, Norwich Research Park, Norwich NR4 7UH, UK. \\ *Present address: Institut Jean-Pierre Bourgin, UMR1318 INRA-AgroParisTech, 78000 Versailles, France. \\ †Present address: The Wellcome Trust Centre for Human Genetics, Roosevelt Drive, Oxford OX3 7BN, UK. \\ ‡To whom correspondence should be addressed. E-mail: caroline.dean@jic.ac.uk
}

\begin{abstract}
Arabidopsis thaliana accessions have adapted to growth in a wide range of climates. Variation in flowering and alignment of vernalization response with winter length are central to this adaptation. Vernalization involves the epigenetic silencing of the floral repressor FLC via a conserved Polycomb (PRC2) mechanism involving trimethylation of $\mathrm{Lys}^{27}$ on histone $\mathrm{H3}$ (H3K27me3). We found that variation for response to winter length maps to cis polymorphism within FLC. A rare combination of four polymorphisms localized around the nucleation region of a PHD-Polycomb complex determines a need for longer cold. Chromatin immunoprecipitation experiments indicate that these polymorphisms influence the accumulation of H3K27me3 in Arabidopsis accession Lov-1, both at the nucleation site and over the gene body. Quantitative modulation of chromatin silencing through cis variation may be a general mechanism contributing to evolutionary change.
\end{abstract}

The molecular basis of natural variation has been defined for relatively few traits. We have focused on natural variation in vernalization, the acceleration of flowering by prolonged cold that aligns flower and seed development with favorable environmental conditions. Vernalization is an inherently quantitative process, with increasing periods of cold giving progressive acceleration of flowering. In A. thaliana this is the result of cell-autonomous stochastic switching of the FLC locus to an epigenetically silenced state, with longer cold increasing the probability of that switch occurring (1). Extensive natural variation exists in A. thaliana accessions for how much cold is required to give complete epigenetic silencing of $F L C(2,3)$. In some accessions, a vernalization period of 4 to 6 weeks of cold gives incomplete FLC silencing; the gene then reactivates after transfer back to warm conditions, and flowering is delayed. This variation has been mapped to several major loci including FLC itself $(3,4)$.

To better understand the molecular basis of this natural variation, we focused on two vernalization-requiring A. thaliana genotypes: Lov-1, an accession collected from the northern range of the species in northern Sweden $\left(62.5^{\circ} \mathrm{N}\right)(5)$, and FRI Col, the reference Columbia accession containing an introgressed active FRIGIDA allele (6). Lov-1 requires 9 weeks of cold to fully saturate the vernalization requirement, thereby epigenetically silencing FLC and enabling activation of the A. thaliana floral promoter FT (Fig. 1A). Partial vernalization of Lov-1 (4 weeks of cold) led to incomplete epigenetic silencing and reactivation of the Lov1 FLC allele upon transfer of plants back to warm conditions, no activation of $F T$, and thus late flowering. In contrast, 4 weeks of cold was sufficient to fully saturate the vernalization requirement in FRI Col, enabling FT activation and early flowering in favorable conditions (Fig. 1A).

Variation in the ability of a fixed length of cold to induce epigenetically stable FLC silencing is therefore a major determinant of flowering time variation in these two genotypes. A substantial fraction of this vari- ation mapped to a large genomic interval including FLC $(3,4)$. We continued to fine-map the quantitative trait locus to an interval of $0.5 \mathrm{Mb}$ containing 182 genes (fig. S1). After experiencing 4 weeks of cold, the flowering of lines carrying the Lov-1 FLC allele introgressed six times into the FRI Col background was significantly delayed (39.5 \pm 1.4 days versus $29.9 \pm 1.8$ days for FRI Col; $n=10$ ). We undertook complementation experiments transforming Col and Lov-1 FLC 10-kb genomic clones into the common background FRI flc-2 and found that transformants carrying the Lov-1 FLC allele flowered significantly later after 4 weeks of cold than did those carrying the Col FLC allele (Fig. 1B). In addition, the Lov-1 FLC expression was reactivated relative to the stable silencing of the Col FLC allele in a Col background (Fig. 1C). Together, these data suggest that cis variation at the Lov-1 FLC allele modulates the length of cold required to achieve the epigenetically stable silencing of FLC, necessary for up-regulation of expression of the floral promoter FT.

The Lov-1 and Col FLC genomic clones used in the complementation experiments differ by 23 single-nucleotide polymorphisms (SNPs) and 16 indels, all in noncoding regions (table S1 and Fig. 2). We generated different combinations of Lov-1 and Col FLC alleles to map the region(s) responsible for the variation in silencing. Ten such mix-andmatch alleles were transformed into FRI flc-2 plants (Fig. 2). To reduce the effect of between-transformant variability, we pooled a large number of seedlings from multiple transformants (between 23 and 49 independent lines) to obtain quantitative FLC expression data before the cold, at the end of the cold, and after transfer back to warm conditions (Fig. 2 and figs. S2 and S3). The minimal regions of the Lov-1 FLC allele sufficient to confer reactivation after 4 weeks of cold were regions b and c, and both together showed a stronger reactivation (Fig. 2, constructs 3, 8, and 9). Only four polymorphisms localize to these regions - two just upstream of the transcription start, the other two at the beginning of intron 1 . These two regions are included in the Polycomb silencing nucleation region, where the PHD-PRC2 (core PRC2 plus three PHD proteins) associates during the cold (7) and where H3K27me3 quantitatively accumulates (1). Inclusion of other Lov-1 sequences, especially the promoter region (region a), enhanced the degree of reactivation (Fig. 2, compare constructs 3 and 6), but the polymorphisms in the promoter were not sufficient by themselves to modulate $F L C$ silencing after cold (Fig. 2, construct 5). The lack of reactivation in construct 4 suggests that other combinations of polymorphisms can also influence the silencing (Fig. 2).

We then looked at the frequency and distribution of the four polymorphisms from regions $\mathrm{b}$ and $\mathrm{c}$ in a selected set of worldwide annual $A$. thaliana accessions requiring different lengths of vernalization (2) (table S2). At positions -121 and +326 relative to the transcription start site, the Lov-1 SNP occurs with high frequency in the populations analyzed. The Lov-1 single-base pair indel at position -56 occurs with medium frequency, whereas the Lov-1 SNP at +598 is rare. The latter is present in only one other accession, Lov-5, which had been found very close to 
the Lov-1 collection site. Only two of the polymorphisms are significantly associated with vernalization response $(-121$ and $+598 ; P<0.01$, Mann-Whitney test; table S3). However, the -121 Lov-1 SNP (the more common type) also associates with flowering time in nonvernalizing conditions. These results suggest that the SNPs can independently influence flowering time in both nonvernalized and vernalized plants, and that it is the combination of specific polymorphisms that leads to the characteristic vernalization response of Lov-1.

Analysis of the phases of vernalization in A. thaliana has shown that the core PRC2 is associated with FLC at all times: before cold, during cold, and after stable silencing has occurred upon return to warm conditions (7-9). The switching of epigenetic states is initiated by coldinduced formation of a PHD-PRC2 complex and by a localized increase in H3K27me3 in the nucleation region. Upon transfer to warm temperature, a subset of the PHD proteins associates with PRC2 over the whole locus, concurrent with a substantial increase in H3K27me3 over the same region. To understand how the polymorphisms that map near this nucleation region might affect the epigenetic silencing, we analyzed H3K27me3 accumulation over Lov-1 FLC at different stages of vernalization (Fig. 3). H3K27me3 levels were found to be much lower over the whole Lov-1 FLC locus before vernalization relative to FRI Col (Fig. 3A), and it took 9 weeks to reach equivalent H3K27me3 levels in the nucleation region in Lov-1 versus 4 weeks in FRI Col. This led to correspondingly lower levels of H3K27me3 over the remainder of the gene after transfer to warm conditions in Lov-1 FLC (Fig. 3B). We then checked whether the lower initial levels of H3K27me3 were also found in FRI Col lines carrying the Lov-1 FLC allele introduced via either introgression or transformation. In both situations, H3K27me3 level was reduced before and after vernalization (Fig. 4), showing that the phenotype was a consequence of cis variation in Lov-1.

How the sequence polymorphisms influence the multiple aspects of H3K27me3 dynamics awaits a fuller understanding of the nucleation process. The four SNPs are not in sequences with homology to known Polycomb response elements or in the region encoding the sense noncoding RNA COLDAIR (10). However, the -121 and -56 SNPs do overlap with the promoter of the sense transcript and the last exon of a spliced variant of COOLAIR, the FLC antisense transcript up-regulated by short cold (11) (fig. S4). We looked at FLC antisense expression, and although we could detect no difference before vernalization, we observed a higher increase in the distally polyadenylated antisense transcript in Lov-1 relative to that in FRI Col after 2 weeks of cold (fig. S5). It is intriguing to speculate that this form of the antisense transcript might antagonize Polycomb complex recruitment (12). This could be due to secondary structure changes caused by the -121 SNP, as suggested by RNAfold predictions (13). The $-121,-56$, and +326 SNPs are within a region previously shown to be required for repression by vernalization (14). The $+598 \mathrm{SNP}$ is within a different functional domain required for maintenance of the silencing (14) and is flanked by two putative binding sites of B3 transcriptional regulators (15). Thus, it is likely that the SNPs influence multiple aspects of the nucleation mechanism.

Cis variation at enhancers or at transcription factor binding sites has played an important role in morphological evolution (16). Our results indicate that cis polymorphism leads to quantitative modulation of a Polycomb silencing mechanism and that this variation alters developmental timing in A. thaliana accessions. The diversity of targets regulated by the Polycomb system presumably constrains changes in the trans factors $(17,18)$. Multiple polymorphisms appear to contribute to the overall effect, with some occurring widely and others infrequently in the population, and the combination of these polymorphisms may be important for the evolution of this trait (19). More generally, our work suggests that quantitative modulation of chromatin silencing mechanisms through cis polymorphisms could be a general mechanism under- lying adaptation of many organisms to changing environments. Evolution of flowering time and vernalization response is an important component of adaptation in A. thaliana (20), and the extent of variation of this trait may well explain the much broader geographical distribution of this species compared to its relatives (21).

\section{References and Notes}

1. A. Angel, J. Song, C. Dean, M. Howard, A Polycomb-based switch underlying quantitative epigenetic memory. Nature 476, 105 (2011). doi:10.1038/nature10241 Medline

2. C. Shindo et al., Role of FRIGIDA and FLOWERING LOCUS C in determining variation in flowering time of Arabidopsis. Plant Physiol. 138, 1163 (2005). doi:10.1104/pp.105.061309 Medline

3. C. Shindo, C. Lister, P. Crevillen, M. Nordborg, C. Dean, Variation in the epigenetic silencing of $F L C$ contributes to natural variation in Arabidopsis vernalization response. Genes Dev. 20, 3079 (2006). doi:10.1101/gad.405306 Medline

4. A. Strange et al., Major-effect alleles at relatively few loci underlie distinct vernalization and flowering variation in Arabidopsis accessions. PLoS ONE 6, e19949 (2011). doi:10.1371/journal.pone.0019949 Medline

5. M. Nordborg et al., The pattern of polymorphism in Arabidopsis thaliana. PLoS Biol. 3, e196 (2005). doi:10.1371/journal.pbio.0030196 Medline

6. S. D. Michaels, R. M. Amasino, FLOWERING LOCUS $C$ encodes a novel MADS domain protein that acts as a repressor of flowering. Plant Cell 11, 949 (1999). Medline

7. F. De Lucia, P. Crevillen, A. M. Jones, T. Greb, C. Dean, A PHD-polycomb repressive complex 2 triggers the epigenetic silencing of FLC during vernalization. Proc. Natl. Acad. Sci. U.S.A. 105, 16831 (2008). doi:10.1073/pnas.0808687105 Medline

8. A. R. Gendall, Y. Y. Levy, A. Wilson, C. Dean, The VERNALIZATION 2 gene mediates the epigenetic regulation of vernalization in Arabidopsis. Cell 107, 525 (2001). doi:10.1016/S0092-8674(01)00573-6 Medline

9. T. Greb et al., The PHD finger protein VRN5 functions in the epigenetic silencing of Arabidopsis FLC. Curr. Biol. 17, 73 (2007). doi:10.1016/j.cub.2006.11.052 Medline

10. J. B. Heo, S. Sung, Vernalization-mediated epigenetic silencing by a long intronic noncoding RNA. Science 331, 76 (2011). doi:10.1126/science.1197349 Medline

11. S. Swiezewski, F. Liu, A. Magusin, C. Dean, Cold-induced silencing by long antisense transcripts of an Arabidopsis Polycomb target. Nature 462, 799 (2009). doi:10.1038/nature08618 Medline

12. C. Chu, K. Qu, F. L. Zhong, S. E. Artandi, H. Y. Chang, Genomic maps of long noncoding RNA occupancy reveal principles of RNA-chromatin interactions. Mol. Cell 44, 667 (2011). doi:10.1016/j.molcel.2011.08.027 Medline

13. A. R. Gruber, R. Lorenz, S. H. Bernhart, R. Neuböck, I. L. Hofacker, The Vienna RNA websuite. Nucleic Acids Res. 36 (web server issue), W70 (2008). doi:10.1093/nar/gkn188 Medline

14. C. C. Sheldon, A. B. Conn, E. S. Dennis, W. J. Peacock, Different regulatory regions are required for the vernalization-induced repression of FLOWERING LOCUS C and for the epigenetic maintenance of repression. Plant Cell 14, 2527 (2002). doi:10.1105/tpc.004564 Medline

15. M. Suzuki, H. H. Wang, D. R. McCarty, Repression of the LEAFY COTYLEDON 1/B3 regulatory network in plant embryo development by VP1/ABSCISIC ACID INSENSITIVE 3-LIKE B3 genes. Plant Physiol. 143, 902 (2007). doi:10.1104/pp.106.092320 Medline

16. S. B. Carroll, Evo-devo and an expanding evolutionary synthesis: A genetic theory of morphological evolution. Cell 134, 25 (2008). doi:10.1016/j.cell.2008.06.030 Medline

17. X. Zhang et al., Whole-genome analysis of histone H3 lysine 27 trimethylation in Arabidopsis. PLoS Biol. 5, e129 (2007). doi:10.1371/journal.pbio.0050129 Medline

18. P. J. Wittkopp, B. K. Haerum, A. G. Clark, Evolutionary changes in cis and trans gene regulation. Nature 430, 85 (2004). doi:10.1038/nature02698 Medline

19. E. J. Hayden, E. Ferrada, A. Wagner, Cryptic genetic variation promotes rapid evolutionary adaptation in an RNA enzyme. Nature 474, 92 (2011). doi:10.1038/nature10083 Medline

20. D. Weigel, Natural variation in Arabidopsis: From molecular genetics to 
ecological genomics. Plant Physiol. 158, 2 (2012). doi:10.1104/pp.111.189845 Medline (Brassicaceae). J. Biogeogr. 29, 125 (2002). doi:10.1046/j.13652699.2002.00647. $\mathrm{x}$

Acknowledgments: We thank all members of the Dean and Howard groups for discussions, and D. Filiault (Gregor Mendel Institute, Vienna) for help with analysis of the SNPs in the worldwide A. thaliana accessions. Supported by a Marie Curie Intra-European Fellowship within the 7th European Community Framework Programme (V.C.), a UK Biotechnology and Biological Sciences Research Council (BBSRC) Core Strategic Grant to the John Innes Centre, and a European Research Council Advanced Investigator grant (C.D.).

\section{Supplementary Materials}

www.sciencemag.org/cgi/content/full/science.1221881/DC1

Materials and Methods

Figs. S1 to S5

Tables S1 to S3

13 March 2012; accepted 7 June 2012

Published online 12 July 2012

10.1126/science.1221881 

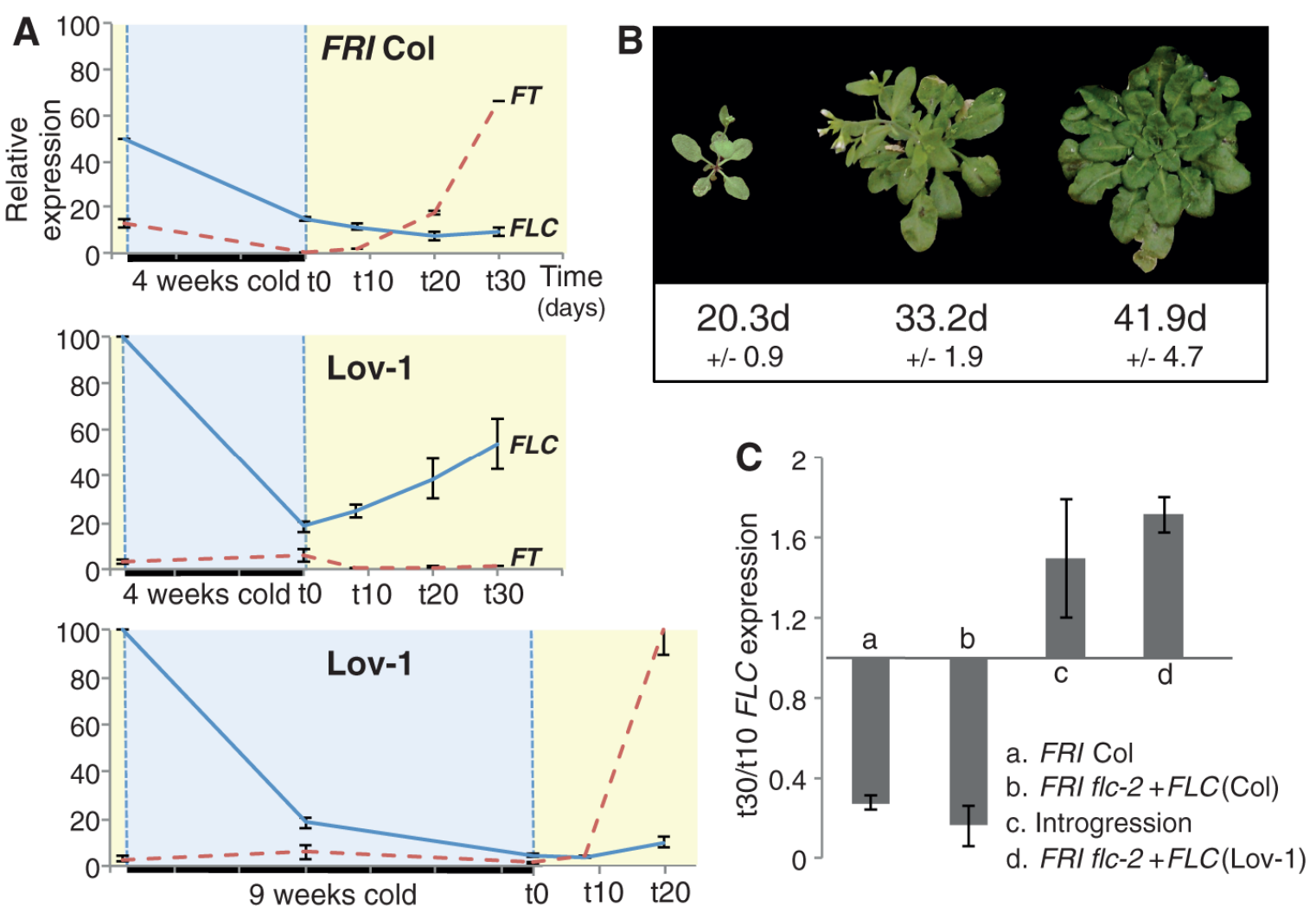

Fig. 1. FLC cis variation delays the vernalization response. (A) FLC and FT expression (solid blue line and red dashed line, respectively) before and after a vernalization treatment of 4 weeks (top and middle) or 9 weeks (bottom) followed by $0,10,20$, and 30 days of warm (t0, $\mathrm{t} 10, \mathrm{t} 20$, and t30). (B) Top: Photograph of FRI flc-2, FRI flc-2 +FLC(Col), and FRI flc-2 $+F L C$ (Lov-1) plants (left to right) vernalized for 4 weeks and then grown in warm long-day conditions for 12 days $(F R I$ flC-2) or 30 days [+FLC(Col), $+F L C(L o v-1)$ ]. Bottom: Average flowering time (d, days to flower) of 10 individuals for each corresponding genotype. (C) FLC expression shown as a ratio of t30/t10 values after 4 weeks of vernalization treatment. A value greater than 1 denotes an increase in FLC expression after return to warm conditions. Introgression is the $F L C(L o v-1)$ genomic region described in fig. S1 in FRI Col. Error bars are SEM from three biological replicates. 


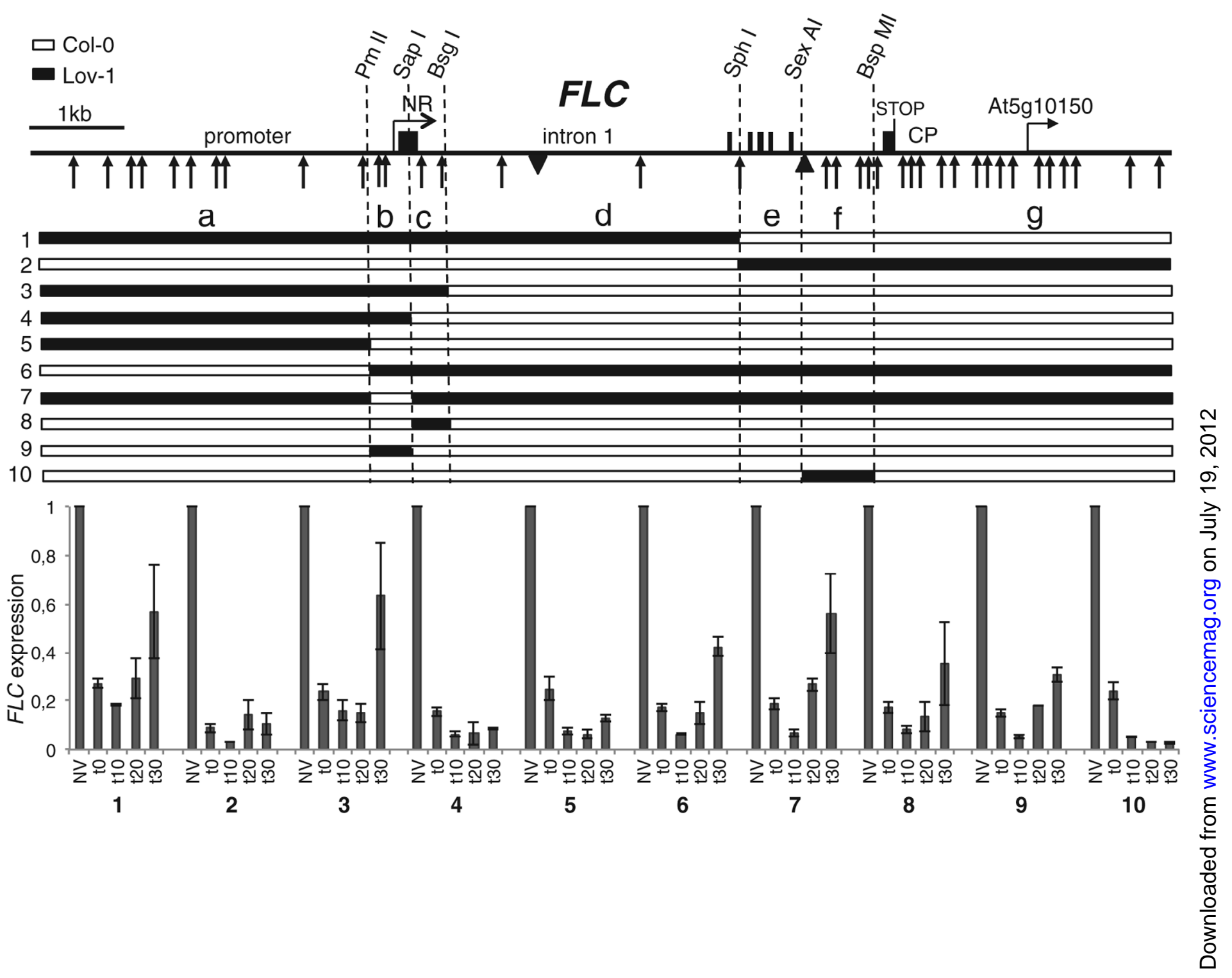

Fig. 2. Mix-and-match $F L C$ alleles define polymorphisms contributing to variation in vernalization response. Top: FLC sequence schematic showing exons (black boxes), positions of SNPs and short indels (arrows), insertion (triangle), deletion (inverted triangle), and restriction sites used for the mix-and-match constructs (with restriction enzymes named). Rows 1 to 10 show composition of the mix-and-match constructs (white, Col sequence; black, Lov-1 sequence). Bottom: FLC expression analysis before (NV) and after a treatment of 6 weeks of vernalization followed by $0,10,20$, and 30 days of warm (t0, t10, t20, and t30) in the 10 different mix-and-match lines. A pool of 23 to 49 independent T2 transformant lines was used for each construct. Error bars are SEM from three biological replicates. 

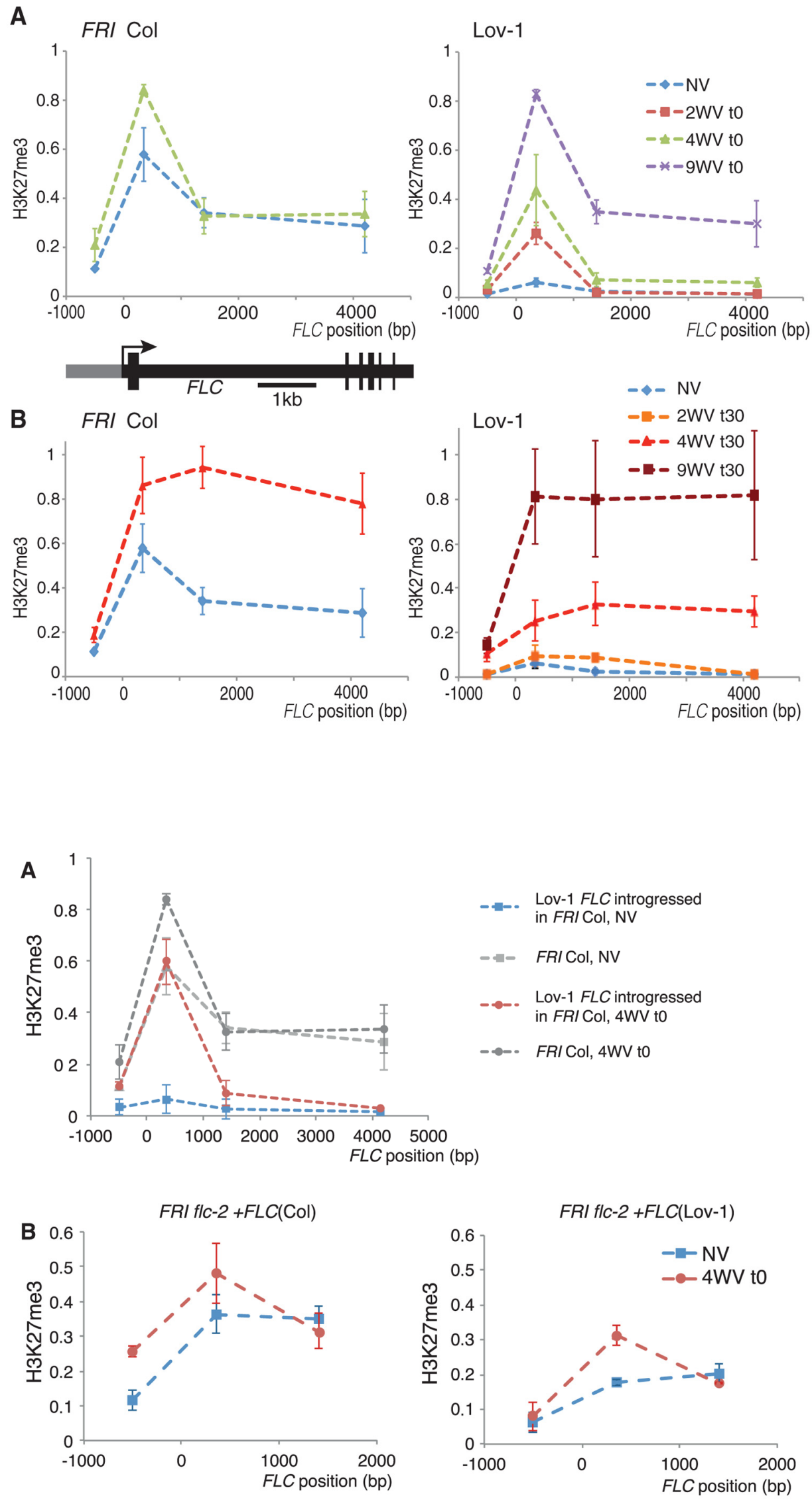

Fig. 3. H3K27me3 accumulation at $F L C$ is delayed in Lov-1. (A) H3K27me3 profile across FLC for Col (left) and Lov-1 (right) plants before vernalization (NV) and after 2, 4, or 9 weeks of cold. (B) H3K27me3 profile across FLC for Col (left) and Lov-1 (right) plants before vernalization (NV) and after 2, 4, or 9 weeks of cold plus 30 days (t30) of growth in warm long-day conditions. Error bars are SEM from three biological replicates.

Fig. 4. Cis variation at $F L C$ results in lower H3K27me3 levels. (A) H3K27me3 profile across FLC for nonvernalized (NV) plants and after 4 weeks of cold (4WV t0) in FRI Col plants or Lov-1 FLC introgressions in FRI Col background. (B) H3K27me3 profile at FLC in regions b and $\mathrm{C}$ for $\mathrm{NV}$ and $4 \mathrm{WV}$ to plants in FRI flc-2 +FLC(Col) pooled T2 transgenics (left) and FRI flC-2 +FLC(Lov-1) pooled T2 transgenics (right). Error bars are SEM from three biological replicates. 